\title{
La docencia de Construcción en el Máster Habilitante en Arquitectura: un reto hacia la profesión
}

\author{
CARMEn GaLÁn Marín \\ Departamento Construcción Arquitectónica 1 \\ Escuela Técnica Superior de Arquitectura \\ Universidad de Sevilla \\ cgalan@us.es \\ ORCID: https://orcid.org/0000-0003-1929-3280 \\ D.O.I.: http://dx.doi.org/10.12795/JDU.2018.i01.14 \\ Pp.: 243-262
}

\section{Resumen}

La presente comunicación se desarrolla como parte del Programa de Formación e Innovación Docente del Profesorado 2018. Se propone el Ciclo de Mejora Docente (CMD) en la asignatura de Construcción e Instalaciones perteneciente al Máster Habilitante en Arquitectura de la Universidad de Sevilla.

En el marco de la docencia de un máster habilitante, la asignatura presenta fundamentalmente un carácter práctico y de habilitación profesional. Esto requiere introducir no ya nuevos contenidos o conceptos, sino su aplicación específica en el campo de la profesión, ejemplificado en el desarrollo de un proyecto de ejecución que el alumno lleva 
a cabo a lo largo del curso, el Proyecto Fin de Carrera (PFC) del estudiante, equivalente al Trabajo Fin de Máster en otras titulaciones.

Durante el diseño del CMD propuesto, se integra la relación entre los contenidos esenciales que debe conocer el estudiante, la realidad en las empresas del sector de la construcción y la toma de decisiones e implementación de recursos en el desarrollo de su proyecto durante las sesiones en el aula. El diseño propuesto ha supuesto un importante avance en esta integración, lo que ha llevado a considerar el éxito en esta primera convocatoria.

Palabras clave: Máster habilitante, Construcción, Arquitectura, Ciclo de Mejora, Innovación Docente.

\section{Interés y Objetivos}

\section{Contexto de la intervención}

La asignatura de Construcción \& Instalaciones forma parte del Máster Habilitante en Arquitectura. El contenido de esta asignatura debe impartirse durante el segundo cuatrimestre en un periodo lectivo de 10 semanas, de equivalente a 5,0 créditos, sirviendo de apoyo y desarrollo de los PFC de cada estudiante. De los 5 créditos, 1 corresponde al profesor de instalaciones, 1 al profesor de Acústica y 3 a la profesora de Construcción. Además de estos créditos la profesora de Construcción tiene otros 4,0 créditos con los mismos estudiantes en la asignatura de PFC, asignatura en la que están implicadas todas las áreas de conocimiento que intervienen en el curso. Se trata a diferencia de otros masters de especialización, fundamentalmente de un master habilitante para la profesión de arquitecto en el que el estudiante desarrolla un proyecto de arquitectura, apoyado por los profesores de diversas áreas de conocimiento en sus respectivos campos.

Los estudiantes para poder matricularse en el máster han debido de cursar las materias troncales del Grado 
en Fundamentos de la Arquitectura relativas a proyectos, construcción, instalaciones, estructuras, mecánica de medios continuos, por lo que han debido adquirir un conjunto de conocimientos básicos que les permitan abordar el contenido propio de un proyecto de ejecución que completará su formación de cara al ejercicio profesional en edificación.

Se trata del tercer año de impartición del máster, primero en el que se hace con más de un grupo para cada asignatura. De acuerdo con las estimaciones de estudiantes a finalizar el grado en el curso anterior, se conforman cuatro grupos de máster habilitante. Es importante señalar la baja matriculación frente a lo esperado para este curso. Ello hace que los grupos sean poco numerosos, en este caso 12 estudiantes de los cuales solo 11 tienen un seguimiento efectivo.

Los objetivos de la asignatura son por una parte aplicar los conocimientos fundamentales de construcción e instalaciones del edificio y preparar al alumno para el ejercicio profesional y por otra fomentar en el alumno su visión global de la profesión de arquitecto en lo que afecta a su responsabilidad y su función en la sociedad.

Para ello, de acuerdo con los Objetivos Generales del Título recogidos en el proyecto docente de la asignatura, se desarrollan los siguientes resultados de aprendizaje:

Conocimiento de los métodos de investigación y preparación de proyectos de construcción.

Aptitud para crear proyectos arquitectónicos que satisfagan a su vez las exigencias estéticas y las técnicas, y los requisitos de los usuarios del edificio respetando límites los impuestos por los factores presupuestarios y la normativa sobre construcción.

Capacidad de comprender la profesión de arquitecto y su función en la sociedad, en particular elaborando proyectos que tengan en cuenta los factores sociales.

La asignatura desarrolla su línea de contenidos planteando una visión global centrada en el diseño de los 
sistemas constructivos de las envolventes del edificio (cerramientos y cubiertas). En cada uno de los sistemas será necesario incluir la definición de materiales y productos llevándolas hasta el nivel de desarrollo necesario en un proyecto de ejecución, esto es planos, memoria constructiva y de cumplimiento de normativa, mediciones y pliego de condiciones.

Arquitectura, técnica, tecnología y construcción se constituyen en las principales líneas argumentales de la asignatura. Las actividades que se definen ella serán las relativas a los sistemas constructivos y de instalaciones del edificio y se desarrollarán dentro del ámbito de los Proyectos Fin de Máster o proyecto Fin de Carrera ideados por cada estudiante.

En el primer cuatrimestre del curso 2017-2018, como parte del Curso de Iniciación del Programa de Formación e Innovación Docente del Profesorado, se realizó un primer ciclo de mejora a nivel de cuarto curso, en la docencia en la asignatura de Taller 5. Durante ese periodo, se trabajó también eminentemente en una actividad práctica en la que el alumno desarrolla un proyecto de arquitectura, apoyado por los profesores de diversas áreas de conocimiento en sus respectivos campos, a nivel de cuarto curso en este caso, e integrando los conocimientos y formaciones propios de dichas áreas.

En el segundo cuatrimestre del curso 217-2018, se toma como punto de partida ese modelo inicial revisándolo y trasladándolo a una docencia mucho más compleja: en el Máster habilitante de arquitectura. Este diseño experimental se realiza durante 20 horas, repartidas en 4 sesiones de 5 horas cada una y centradas en dos de los temas que forman parte de la asignatura. 


\title{
Dificultades encontradas en el aprendizaje de la asignatura
}

\author{
El Máster se divide en tres módulos con el siguiente \\ esquema:
}

1. Técnico (10 ECTS): Estructuras y Cimentaciones 05 ECTS + Construcción e Instalaciones 05 ECTS.

2. Proyectual (20 ECTS): Proyectos Avanzados en Arquitectura 14 ECTS + Planeamiento y Proyectos Urbanos 06 ECTS

3. Trabajo Fin de Máster: El PFC es, de acuerdo al plan de estudios, tanto una asignatura como un trabajo académico que llevará a cabo el estudiante con apoyo docente y que tiene por objetivo que éste culmine un proceso de adquisición de competencias y conocimientos en los estudios de máster, hasta alcanzar las competencias necesarias para gestionar los procesos que requieren los diversos tipos de proyectos arquitectónicos.

Los trabajos académicos PFG/PFC, por la propia definición de la Orden Ministerial como Título habilitante, sólo podrán ser evaluados una vez superadas las demás asignaturas de la titulación, en este caso la asignatura de Construcción e Instalaciones.

Se requiere también que el estudiante comprenda; los problemas de la concepción estructural, de construcción y de ingeniería vinculados con los proyectos de edificios así como las técnicas de resolución de estos; y las relaciones entre las personas y los edificios y entre éstos y su entorno, así como la necesidad de relacionar los edificios y los espacios situados entre ellos en función de las necesidades y de la escala humanas.

El máster habilitante (Plan 2012) se encuentra aún en su tercer año de andadura, no existiendo conceptos equivalentes para las distintas asignaturas en planes de estudios anteriores. Es por ello que el reto principal de la asignatura, como disciplina técnica, es lograr que los estudiantes 
integren los conceptos aprendidos en el grado de manera que sean capaces de aplicarlos en la definición constructiva de su proyecto, de forma gráfica y técnica. A menudo, el problema que nos encontramos es de carencias u olvido de conceptos básicos pertenecientes a cursos anteriores y sobre todo de la imposibilidad de aplicar en la práctica lo aprendido.

Asimismo, el otro principal problema nace de la propia la necesidad de desarrollar la asignatura como apoyo al PFC del estudiante. Esto hace que el mayor o menor grado de desarrollo del mismo al iniciar la docencia de la asignatura en el segundo cuatrimestre sea clave a la hora de asimilar las cuestiones planteadas en la asignatura: si un estudiante aún está intentando dar respuesta con su proyecto a cuestiones tales como implantación, alineaciones, volúmenes, programa de usos, difícilmente podrá centrarse o mostrar interés en cuestiones relativas a la formalización del cerramiento o la cubierta del edificio. Para ello los tiempos del proyecto son fundamentales: sólo si un proyecto está definido a nivel de proyecto básico (que conlleva definir las características generales del edificio) al final del segundo cuatrimestre (enero), tiene sentido afrontar las cuestiones relativas al proyecto de ejecución y contar con las asignaturas de apoyo al mismo, "Estructuras y Cimentaciones" y "Construcción e Instalaciones" dispuestas en el segundo cuatrimestre.

La organización de horarios con sesiones de 5 horas de duración hace que sea necesario plantear actividades distintas a lo largo de la mañana para evitar descenso en el nivel de atención del estudiante, evitar la monotonía e impulsar el aprendizaje.

Frente a esos problemas, se han planteado los principios didácticos sobre los que se basa el diseño experimental propuesto en la asignatura. 


\section{Diseño del CMD}

\section{Principios didácticos argumentados que han guiado el Ciclo de Mejora.}

Es necesario fomentar que los estudiantes puedan desarrollar la capacidad de razonamiento autónomo y toma de decisiones, por tratarse de un máster que los habilita para ejercer la profesión de arquitecto con las responsabilidades y atribuciones que marca la Ley de Ordenación de la Edificación Ley 38/1999, de 5 de noviembre. Por ello puede decirse que el objetivo docente principal es la capacitación del alumnado en competencias profesionales, como potenciales responsables del diseño del edificio.

Se trata de una asignatura donde especialmente se desarrollan aspectos procedimentales necesarios para abordar el desarrollo constructivo de la redacción de un proyecto de ejecución de una edificación proyectada por el estudiante. Así pues, podría definirse como aprendizaje mediante la resolución de problemas y por tanto fomentando el aprendizaje activo.

El cambio en el modelo metodológico que se propone se va a realizar en cuatro sesiones, acompasando dos asignaturas e incorporando profesionales del sector que, intervendrán en las sesiones dándoles una perspectiva claramente profesional y por otra parte utilizando el formato taller con las exposiciones de los estudiantes que tendrán que defender y argumentar las características de determinados sistemas constructivos de su proyecto.

Por todo ello el planteamiento docente del ciclo intentará situar al estudiante en el proceso de toma de decisiones habitual en el entorno profesional, fomentando que investigue sobre sistemas constructivos, complemente conocimientos mediante asesores externos (empresas del sector), tome decisiones confiando en sus propias capacidades, valore actuaciones y defina al máximo los sistemas 
constructivos proyectados y prescritos para su edificio (el problema planteado). Los principios didácticos en los que se basa el diseño de la asignatura consideran:

- Mayor participación del estudiante en su propia formación: Para ello, la docencia se presenta como respuesta a cuestiones que necesariamente debe plantearse para el desarrollo de su propio proyecto del mismo modo que se realiza en la práctica profesional.

- Que los estudiantes lleven a cabo un proceso reflexivo, crítico y propositivo que les permita desarrollar procesos de análisis y síntesis que integren las condiciones de la propuesta y sus necesidades, incorporando referencias interdisciplinares como base para la definición de los criterios de intervención en propuestas arquitectónicas.

- Clases que deben ser más participativas: Se buscará en todo momento la participación del estudiante en el aula mostrando a los demás mediante puestas en común su propio proceso de búsqueda de información y resolución de problemas de forma que el camino recorrido (y no sólo los resultados) por los más avanzados pueda servir de incentivo para aquellos que se encuentran más rezagados.

- Relacionar contenidos con otras asignaturas de interés: Se facilitará el recuerdo de lo ya aprendido en cursos del grado con enlaces, referencias, apuntes y resúmenes como complemento a las clases.

\section{Modelo metodológico actual:}

En el planteamiento docente actual cada tema se imparte en jueves, a modo de clase magistral en la primera mitad de la mañana y en la segunda mitad se realizan correcciones individuales con cada uno de los proyectos de los estudiantes. 
Tabla 1. Correcciones individuales según Secuencia de Actividades clase de cubiertas.

\begin{tabular}{|r|l|r|}
\hline & $\begin{array}{l}\text { CLASE 1: CUBIERTAS } \\
\text { SECUENCIA DE ACTIVIDADES. }\end{array}$ & $\begin{array}{r}\mathbf{5} \text { horas } \\
2.5+2.5 \mathrm{~h} \\
300 \text { minutos }\end{array}$ \\
\hline $\mathrm{S}$ & Llegada a la clase. Saludos. & 15 minutos \\
\hline $\mathrm{E}$ & Comentarios previos sobre el estado de los proyectos. & 15 minutos \\
\hline $\mathrm{T}$ & Clase teórica sobre tema específico global orientados al PFC & 30 minutos \\
\hline $\mathrm{P}$ & $\begin{array}{l}\text { Comienzo de correcciones individuales con cada uno de los proyectos de los } \\
\text { estudiantes }\end{array}$ \\
\hline $\mathrm{C}$ & $\begin{array}{l}\text { Se interrumpe a veces para hacer comentarios generales sobre las } \\
\text { correcciones que interesan a todos los estudiantes }\end{array}$ \\
\hline & $\begin{array}{l}\text { Continuación de correcciones tutorizadas individuales con cada uno de los } \\
\text { estudiantes. }\end{array}$ & 5 minutos \\
\hline $\mathrm{CF}$ & $\begin{array}{l}\text { Comentarios generales de cara a la sesión del próximo día y continuación } \\
\text { del trabajo. }\end{array}$ & 120 minutos \\
\hline & \multicolumn{1}{|c|}{ Total } \\
\hline
\end{tabular}

\section{Modelo metodológico y secuencia de actividades programadas para su aplicación.}

El ciclo se desarrollará en dos sesiones, la primera de ellas específicamente dedicada a las cubiertas de los edificios y en la segunda sesión a una tipología específica de envolvente vertical concretamente cerramientos realizados con muros cortina de vidrio.

La teoría estará intercalada entre dos momentos prácticos $(P-T-P)$, de manera que los estudiantes hagan una primera propuesta en la clase de PFC de martes de, por ejemplo, las cubiertas de su proyecto. El jueves en una primera parte se les aporte la información teórica (o técnica) que les corrija errores y les ayude a mejorar sus propuestas y tras un debate en formato taller, finalmente entregarán una versión mejorada.

Se propone un modelo metodológico en la Figura 1 en el que se diferencian cuatro fases de trabajo paralelas al proceso de aprendizaje que se espera. El desarrollo docente se apoya en el Proyecto Fin de Carrera del estudiante proponiendo para éste un sistema constructivo cerramiento o cubiertas. Se utilizan para ello las 5 horas de esa semana de PFC y las 5 de la asignatura de Construcción e Instalaciones. Durante la revisión del proyecto se 
planteará al estudiante una secuencia de preguntas inmediatas a partir de las cuales se extraen las cuestiones claves a resolver en su proyecto. La resolución de nuevas incógnitas supondrá avanzar de forma encadenada en la profundización y desarrollo de los sistemas constructivos, productos de construcción y detalles necesarios para llevarlos a cabo en su proyecto de ejecución.

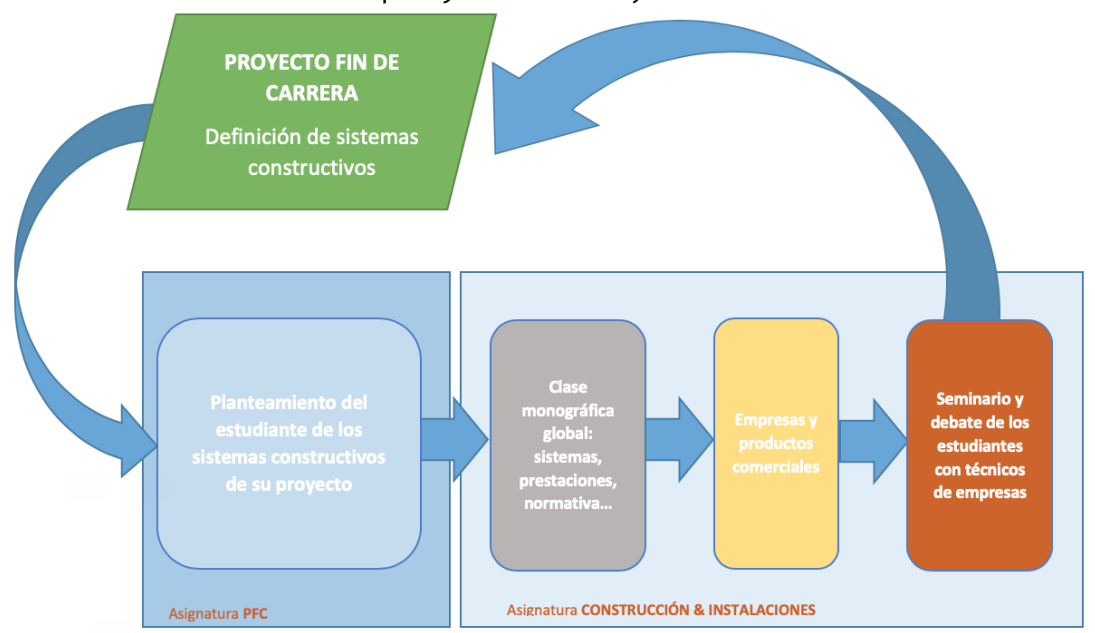

Figura 1. Nuevo Modelo Metodológico propuesto.

En este modelo, se incorpora un mayor control de los tiempos en el aula y dedicación en la misma a las actividades prácticas manteniendo la curiosidad del estudiante y su implicación en la resolución de cuestiones sobre su proyecto del modo más cercano posible a futura práctica profesional. La Tabla 2 recoge la Secuencia de Actividades prevista.

Jornadas de Formación e Innovación Docente del Profesorado | № 1 (2018) Esta obra se distribuye con la licencia Creative Commons 
Tabla 2. Secuencia de Actividades clase de cubiertas.

\begin{tabular}{|c|c|c|}
\hline & SECUENCIA DE ACTIVIDADES. & 10 horas \\
\hline & CLASE DE PFC (martes) & 5 horas \\
\hline $\mathbf{S}$ & $\begin{array}{l}\text { Llegada a la clase. Saludos. Entrega del avance de su proyecto denominado "Hito 2: } \\
\text { Definición de sistemas constructivos y estructurales" }\end{array}$ & 20 minutos \\
\hline $\mathbf{S}$ & $\begin{array}{l}\text { Presentación de la actividad. Explicación del trabajo en grupos. Entrega de las } \\
\text { preguntas encadenadas. Comienzo del trabajo en grupos. }\end{array}$ & 10 minutos \\
\hline \multirow[t]{2}{*}{ G } & Desarrollo del trabajo en grupos. & 120 minutos \\
\hline & Descanso & 30 minutos \\
\hline G & Desarrollo del trabajo en grupos. & 120 minutos \\
\hline \multirow[t]{3}{*}{$\mathbf{t}$} & Tutorización por grupos & \\
\hline & Total & 5 horas \\
\hline & CLASE DE CONSTRUCCIÓN E INSTALACIONES (jueves) & 5 horas \\
\hline \multirow[t]{2}{*}{$\mathbf{T}$} & $\begin{array}{l}\text { Clase monográfica del profesor tipologías y sistemas de cubiertas orientada al PFC. } \\
\text { Requerimientos según normativa y clasificación global de sistemas disponibles. }\end{array}$ & 85 minutos \\
\hline & Descanso & 30 minutos \\
\hline $\mathbf{E}$ & $\begin{array}{l}\text { Seminario de presentación de productos y sistemas de empresa de cubiertas e } \\
\text { impermeabilizaciones. Planteamiento de sus posibilidades y sistemas constructivos. } \\
\text { Se aportarán muestras y catálogos con especificaciones técnicas. }\end{array}$ & 90 minutos \\
\hline e+d & $\begin{array}{l}\text { Exposición por parte de cada estudiante, de los sistemas constructivos de cubiertas } \\
\text { utilizados en su proyecto. Discusión sobre los detalles de la misma con los técnicos } \\
\text { de las empresas de productos de construcción que intervendrán como asesores. A } \\
\text { modo de moderador, el profesor dirigirá el debate y preguntará sobre aquello que } \\
\text { estime que ha quedado sin comentar. }\end{array}$ & 120 minutos \\
\hline CF & Comentarios generales y continuación del trabajo. & 5 minutos \\
\hline \multirow[t]{3}{*}{ te } & Encuesta/test & \\
\hline & Total & 5 horas \\
\hline & Total ciclo de mejora & 10 horas \\
\hline
\end{tabular}

Como detonante y punto de partida del modelo metodológico propuesto, se recoge a los estudiantes es día en la clase de PFC (martes) el avance de su proyecto denominado "Hito 2: Definición de sistemas constructivos y estructurales". Tras ello se entrega a los estudiantes una seria de preguntas encadenadas o problemas a las que deben enfrentarse en grupos. El cuestionario se analiza en detalle en el apartado correspondiente.

En el aula se distribuye el tiempo entre el trabajo de los estudiantes en grupos (G) y la atención o tutorización personalizada del profesor (T). En casa el estudiante deberá aplicar la problemática surgida al proyectar y resolver la cubierta a su proyecto concreto. En la sesión siguiente (jueves de la misma semana) se realiza inicialmente una clase monográfica (M) que recoge y estructura los conceptos sobre los que han trabajado los estudiantes. Tras ello se presenta a una empresa del sector que mostrará los 
productos $(P)$ y sistemas de que dispone en el mercado para resolver ciertas tipologías de la envolvente del edificio. Tras ello se hará una puesta en común de todos los estudiantes (C) con una exposición individual previa de su proyecto de forma resumida y global, e irán planteando (guiados por la profesora y asesorados por los técnicos de la empresa que participa), la viabilidad de su proyecto y las posibles soluciones con productos y sistemas reales, entre otros de la empresa propuesta.

Del mismo modo se desarrolla la clase 2 del ciclo: seminario sobre fachadas de vidrio/muro cortina, con la misma secuencia de actividades anterior pero cambiando el objeto de estudio.

\section{Mapa de contenidos y problemas o preguntas clave.}

El mapa de contenidos y problemas que se muestra en la página siguiente pretende poner en relación los conocimientos relativos al área reflexión sobre los contenidos de aprendizaje y las preguntas o problemas claves que hay que resolver en las partes de los proyectos implicadas en el ciclo de mejora de construcción que los estudiantes necesitarán implementar en su proyecto.

Para profundizar y relacionar estos conceptos se solicitó a los estudiantes en las primeras sesiones del curso ya en el mes de enero que trabajaran en grupos sobre una serie de edificios construidos, ejemplos propuestos. En el momento de desarrollo del ciclo, jueves 10 y 24 de abril de 2018, el problema de los estudiantes es ya desarrollar su propio proyecto. 


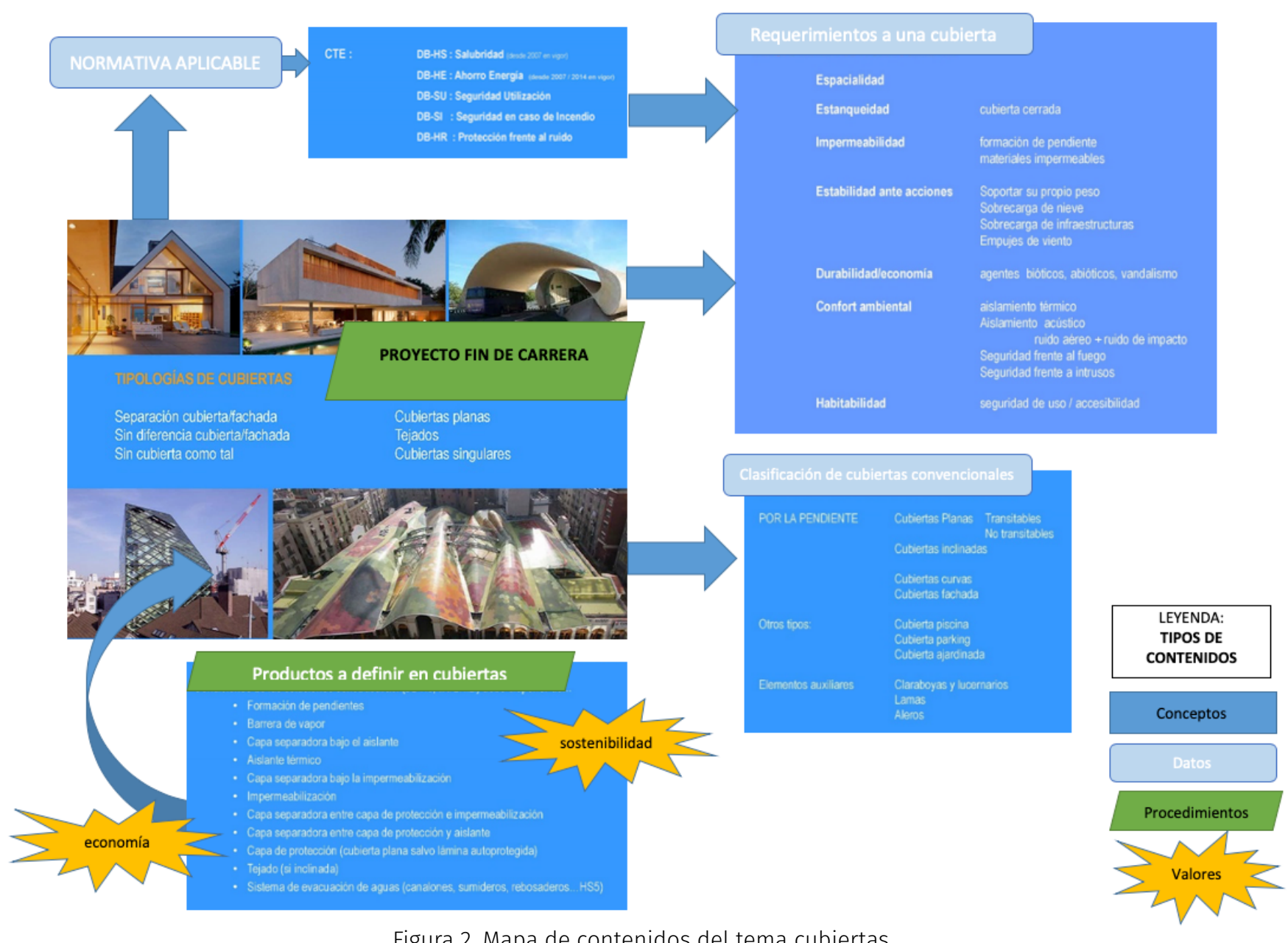

Figura 2. Mapa de contenidos del tema cubiertas.

Jornadas de Formación e Innovación Docente del Profesorado | № 1 (2018)

(c) Esta obra se distribuye con la licencia Creative Commons

(c) $(7) \Theta$ Reconocimiento-NoComercial-SinObraDerivada Internacional (CC BY-NC-ND 4.0.) 


\section{Aplicación del CMD}

\section{Valoración sobre el desarrollo de las sesiones}

Respecto a las clases anteriores y como también ocurrió en el CMD desarrollado en 2017 en la asignatura de taller, se ha superado la dificultad encontrada sobre la necesidad de generar el interés en el estudiante por trabajar en el aula y no solo escuchar.

Por otra parte, la implicación de los estudiantes es muy alta al tratarse de su propio proyecto. Las actividades planteadas en grupo han favorecido el re-enganche de los estudiantes que tienen el planteamiento del proyecto más atrasado.

Las preguntas sobre las que trabajan en la primera sesión del martes les han servido a modo de salvavidas o más bien de "navegación de cabotaje" asegurándose de que no perdían la tierra firme de vista ni un solo momento.

Resulta el jueves complicado mantener la limitación de tiempo de cada parte del programa. Una de las empresas implicadas alarga excesivamente su tiempo de exposición. Los estudiantes en su presentación muestran imágenes y también les pido que sus dibujos de detalle los lleven a la pizarra con lo que se provoca una clase muy dinámica donde todos están centrados en lo que estamos trabajando. Se respira interés y armonía entre estudiantes, técnicos de empresa y docente.

Al final de la clase aún se quedan muchos estudiantes deseando seguir charlando con el profesor y con los técnicos de las empresas, sobre detalles que no ha dado tiempo. Sus comentarios al finalizar son muy positivos, me dicen cosas como "distinto", "más dinámico", "ejemplos muy ilustrativos".

Sus comentarios en general son muy positivos. Para poder apreciarlo mejor se les ha pedido al final rellenen 
una encuesta de la que se muestra un ejemplo en la página siguiente.

Entre los aciertos del CMD propuesto indicar que se han conseguido unas jornadas/clases más interactivas con la participación de todos y los estudiantes han podido compartir y enriquecerse con la experiencia de los demás.

\section{Evaluación del progreso de aprendizaje}

Tras la realización del Ciclo de Mejora se procedió a comparar los resultados no ya de un cuestionario previo frente a otro posterior sino de la entrega de los estudiantes el mismo día de inicio del ciclo (hito 2 del curso) y la entrega preparan durante el ciclo y que se proyecta y sobre la que se discute en la clase.

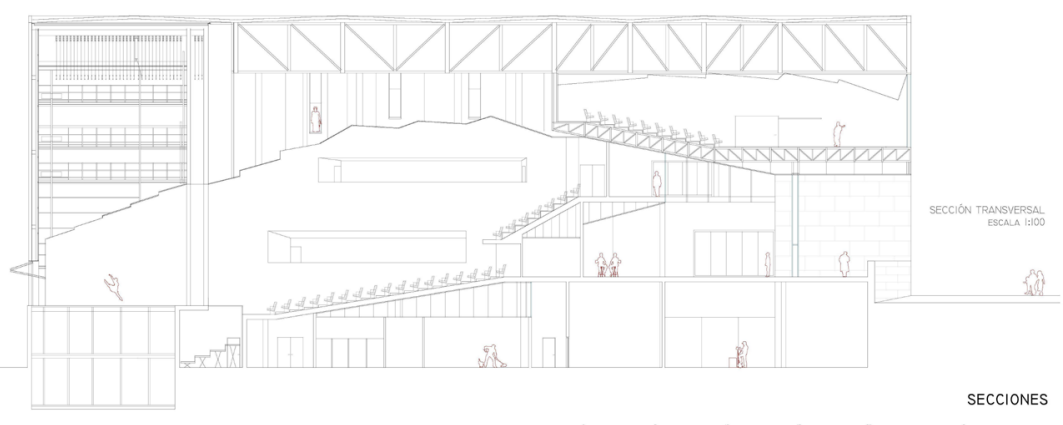

Figura 3. Nivel de definición del proyecto antes del CM. Estudiante Francisco valdayo.

Son muy significativos, en todos los estudiantes, los avances habidos dentro de la definición constructiva del proyecto de cada uno de ellos, como muestran las figuras 6 a 8 . Al tratarse de proyectos individuales de los estudiantes es muy complicado plantear escaleras de conocimiento del grupo. Por ello, no ha sido posible plantear un diagrama en escalera que muestra los distintos niveles en la progresión del aprendizaje en los que puede situarse al estudiante sino más bien contemplar el notable cambio en todos ellos. Desde el punto de vista técnico la 
información gráfica presentada tras el ciclo es mucho más completa y precisa.

Los estudiantes han mostrado en todo momento un buen nivel de motivación y participación. Además la dinámica ha permitido integrar a aquellos cuyas carencias en el desarrollo del proyecto en las fases anteriores (aún no completadas y sustentadas en otras asignaturas) les impiden tomar las decisiones aún en este punto. Creo que los estudiantes han identificado claramente los conceptos que deben trabajar conmigo como profesora en su proyecto.

En las sesiones se han visto en el aula momentos de concentración y trabajo y la sensación de aprovechar intensamente el tiempo. El Ciclo de Mejora ha permitido romper la dinámica habitual de la clase con correcciones específicas individuales tutorizadas, a la que están acostumbrados los estudiantes. Además les ha obligado a realizar un trabajo contra reloj poco frecuente en clase haciéndola más interesante. También les ha dado la oportunidad de participar y expresar sus propias ideas.

En cuanto a las dificultades encontradas, la primera de ellas fue la necesidad de que todos los estudiantes participasen. La dinámica ha conseguido romper un poco la distancia entre aquellos proyectos más avanzados y aquellos que aún están en fase de toma de decisiones. El bajo número, 11 estudiantes, favorece esto pero creo que esta dinámica no sería factible con tanta disparidad de niveles si fuesen más.

La tercera dificultad fundamental fue la gestión del tiempo. Es absolutamente necesario controlar la duración de las actividades de forma constante en la sesión. Lo más difícil de gestionar es el tiempo de la puesta en común. 


\section{Evaluación del CMD aplicado}

\section{Valoración de la experiencia}

A las cuestiones planteadas, las respuestas confirman el interés despertado en los estudiantes.

Con respecto al enunciado o cuestionario entregado la primera clase, el $100 \%$ de los alumnos comentaba que las preguntas trabajadas en la primera clase, le habían servido como guía para los aspectos a definir y diseñar en su cubierta. En muchos casos añadían "cuestiones a las que aún no me había enfrentado"

A nivel de curso de máster, se considera necesaria la breve teoría explicada por el profesor en palabras de los estudiantes "para refrescar conocimientos" aunque un 15\% de los alumnos la hubiesen preferido "un poco más extensa para poder recordar conceptos con más facilidad".

La intervención de los especialistas de cubiertas de empresas se considera "muy útil" ya que la utilización de un determinado producto de construcción "influye de forma crucial en el diseño". El $100 \%$ de los alumnos menciona que resuelven dudas en cuanto al funcionamiento de los distintos sistemas, y valoran su opinión y experiencia a la hora de adoptar una u otra solución en su proyecto. Así mismo se valora su exposición previa de la "amplia gama de productos disponibles en el mercado y la gran versatilidad que existe a la hora de resolver" "aportando a la vez alternativas y soluciones".

Se menciona que aportan un punto de vista diferente del habitual en el aula y enriquece mucho frente a la simple lectura de un catálogo. Otros comentan que "hoy en día existen multitud de productos y muchos parecen iguales. Saber cuál elegir en cada caso es esencial".

El arquitecto a la hora de realizar el diseño de un edificio es el prescriptor de los equipos productos y sistemas que se colocan en una obra. Por ello los alumnos necesitan 
enfrentarse en sus proyectos a la definición de especificaciones técnicas y dimensionales de los materiales y productos utilizados.

Destacan comentarios condicionados desde su enfoque previo de la profesión como que "me gusta también que tengan en cuanta tanto el diseño del arquitecto, creía que la realidad era muy distinta" y "te hablan en lenguaje propio de la profesión". Se valora también que aporten un "punto de vista ajeno al ámbito académico".

El 100\% de los asistentes solicita, entre las posibles mejoras, realizar más clases de este tipo aplicadas a otros sistemas constructivos: fachadas ventiladas o con soluciones pesadas por ejemplo. En el caso de la empresa de muros cortina se menciona también que su gama de productos en más limitada limitándose a más sistemas convencionales tipo stick y querrían poder contar con empresas dedicadas a proyectos "menos convencionales".

La sensación general es muy satisfactoria, corroborada por los propios comentarios de los estudiantes. Los profesores también me han expresado su satisfacción y la intención de trasladar la experiencia a los otros grupos de la asignatura que trabajan coordinadamente con nosotros.

\section{Conclusiones finales}

Tras entrega de primera convocatoria, se observa que efectivamente el curso global presenta a una baja tasa de éxito pese a la implementación del ciclo de mejora. Tan sólo cuatro de los once alumnos del grupo han realizado la entrega final de la asignatura al llevar como se observó al principio del cuatrimestre, un gran retraso sobre lo previsto en cuanto a la definición del proyecto básico. Sólo uno de los once ha entregado el Proyecto Final de Carrera en junio. Es necesario por lo tanto hacer hincapié en cursos posteriores en el cumplimiento del calendario de tomas de decisiones de los proyectos de los alumnos con respecto a las otras asignaturas. 
Este bajo porcentaje de éxito contrasta con la positiva actitud de los estudiantes que, asistiendo a clase hasta el final, han seguido toda la dinámica establecida y como resultado todos muestran grandes avances en la definición de sus proyectos frente a la situación de marzo.

También se ha observado un descenso significativo en la petición de tutorías "veraniegas" por parte de los estudiantes implicados (que plantean entregar en septiembre) lo que refuerza que la metodología aplicada para el aprendizaje les permite tomar sus propias decisiones a partir de actividades en el aula y en consecuencia la resolución de cuestiones que lo acercan a su práctica profesional.

Respecto al desarrollo de la entrega de la asignatura, se ha detectado la dificultad del estudiante para elaborar la memoria descriptiva y justificativa de cumplimiento de normativa como parte del proyecto de ejecución completo por lo que, en clase, se incluirán actividades que revisen este aspecto.

En cualquier caso, dadas las dificultades para representar los resultados de aprendizaje en una escalera de progresión del aprendizaje, para el próximo curso y CMD, se propone llevar a cabo un desarrollo más específico de las escaleras de aprendizaje.

\section{Agradecimientos.}

La autora quiere agradecer a los técnicos de las empresas SIKA SAU y Cortizo su disponibilidad y presencia en el aula, su entusiasta colaboración en el desarrollo de esta experiencia, sin la cual no habría sido posible, atendiendo en todo momento a los alumnos y brindándoles trato profesional. 


\section{Referencias bibliográficas}

Bain, K. (2004). Traducción Barberá, O. (2008). Lo que hacen los mejores profesores universitarios. Universidad de Valencia. España: PUV.

Bahón, J. (2016). Laboratorio Internacional de Innovación y Coaching Educativo. Recuperado de https://www.tuinnovas.com.

Finkel, D. Traducción Barberá, O. (2008). Dar clases con la boca cerrada. Valencia. España: Universidad de Valencia.

Libro de resúmenes del 1 Congreso Internacional de Innovación y Tendencias Educativas. INNTED (2017). Innovación y Tendencias Educativas. Sevilla. España: Universidad de Sevilla.

Porlán, R. (2017). Enseñanza Universitaria. Como mejorarla. Universidad de Sevilla. España: Ediciones Morata.

Proyecto de Innovación Docente II Plan propio de Docencia. Departamento de Estructuras de la Edificación e Ingenieria del Terreno (Convocatoria 2016). El aprendizaje de conceptos umbral mediante tics en un entorno multidisciplinar. Universidad de Sevilla.

Proyecto de Innovación Docente III Plan propio de Docencia. Departamento de Construcción Arquitectónica 1 (Convocatoria 2017). Tutorización de las horas de aprendizaje no presencial mediante tics en disciplinas técnicas. Universidad de Sevilla.

Jornadas de Formación e Innovación Docente del Profesorado | № 1 (2018) Esta obra se distribuye con la licencia Creative Commons 